\title{
Review
}

\section{Baculoviruses and apoptosis: the good, the bad, and the ugly}

\author{
RJ Clem ${ }^{*, 1}$ \\ ${ }^{1}$ Division of Biology, Kansas State University, Manhattan, Kansas, USA \\ * Corresponding author: R Clem, 232 Ackert Hall, Division of Biology, Kansas \\ State University, Manhattan, KS 66506, USA Tel: +1 785 532-3172; \\ Fax: +1 785 532-6653; E-mail: rclem@ksu.edu
}

Received 30.10.00; accepted 4.12.00

Edited by J Hardwick

\begin{abstract}
Since 1991, when a baculovirus was first shown to inhibit apoptosis of its host insect cells, considerable contributions to our knowledge of apoptosis have arisen from the study of these viruses and the anti-apoptotic genes they encode. Baculovirus anti-apoptotic genes include $p 35$, which encodes the most broadly acting caspase inhibitor protein known, and iap (inhibitor of apoptosis) genes, which were the first members of an evolutionarily conserved gene family involved in regulation of apoptosis and cytokinesis in organisms ranging from yeast to humans. Baculoviruses also provide an ideal system to study the effects of an apoptotic response on viral pathogenesis in an animal host. In this review, I discuss a number of interesting recent developments in the areas of apoptotic regulation by baculoviruses and the effects of apoptosis on baculovirus replication and pathogenesis. Cell Death and Differentiation (2001) 8, 137-143.
\end{abstract}

Keywords: baculovirus; apoptosis; p35; iap; caspase; insect immunity

Abbreviations: AcMNPV, Autographa californica multicapsid nucleopolyhedrovirus; iap, inhibitor of apoptosis; BIR, baculovirus IAP repeat; BIRP, BIR-containing protein

\section{Introduction}

Many people are of the opinion that all viruses are 'bad' or harmful. But as all virologists know, there are some examples of viruses that are quite beneficial to humankind. One such example is the family of viruses known as baculoviruses. Baculoviruses are large DNA viruses that infect arthropods and are ubiquitous in the environment. The several hundred baculoviruses that have been described in the literature are highly specific for closely related groups of insects or crustaceans. Because of their restricted host range and their ability to kill insects, baculoviruses have been used in biological control of pest insects, and there is great interest in further improving them for this use. In the world of biomedical research, the baculovirus expression system is a workhorse for foreign gene expression and is one of the most widely used eukaryotic expression systems today. The ability of baculoviruses to enter almost any kind of cell but only replicate in certain insect cells has also sparked interest in their use as human gene therapy vectors.

But in addition to all of these wonderful benefits that have arisen from studying baculoviruses, another discovery stemming from baculovirus research that has had an impact on basic biomedical research was the discovery of the ability of baculoviruses to control apoptosis in their host insect cells and the identification of baculovirus genes that are responsible for this ability. The use of baculovirus antiapoptotic genes as tools to dissect apoptotic pathways has greatly increased our understanding of the processes involved in regulating apoptosis. The first such gene identified, $p 35$, encodes the most widely acting antiapoptotic gene currently known; it is the only known gene that has anti-apoptotic activity in all three of the major genetic systems used to study cell death, the nematode, the fly, and the mouse, despite the fact that cellular homologs of $p 35$ have not been discovered yet. In addition, the first homologs of the increasingly important family of iap genes were also discovered in baculoviruses, and the study of baculovirus iap genes continues to contribute to our understanding of apoptosis regulation. Finally, our understanding of basic innate immunity is also being benefited by studies on how baculoviruses regulate apoptosis in their insect hosts and the consequences for viral replication and pathogenesis if they fail to do so.

This review is organized from the perspective of the insect host: the ability of host cells to sense viral infection and commit suicide by triggering apoptosis is good from the host's perspective, because killing off a small number of initially infected cells helps the insect fight off the infection. On the other hand, the ability of the virus to block the host cell's attempt at suicide is bad for the host, because it results in successful infection and death of the host (which is where the ugly part comes in, as we will see). Of course, the converse is true from the point of view of the virus. Baculoviruses, like many other viruses, must find ways to block the apoptotic response of their host cells in order to be productive parasites, and in this they have succeeded admirably.

\section{The good: how do baculoviruses induce apoptosis?}

The same baculovirus that is widely used for foreign gene expression, Autographa californica multicapsid nucleopolyhedrovirus (AcMNPV), was one of the first viruses shown to influence host cell apoptosis. ${ }^{1}$ AcMNPV replicates in larvae 
(caterpillars) of several species of the insect order Lepidoptera, which includes moths and butterflies. A spontaneous mutant of AcMNPV known as the annihilator mutant was found to induce apoptosis in the lepidopteran insect cell line SF-21. When the mutation in the annihilator mutant was mapped, it was found to contain a deletion in the p35 gene, ${ }^{1}$ implying that the $p 35$ gene was able to prevent the apoptotic response normally induced by AcMNPV infection in this cell line. Despite significant progress in our understanding of how baculoviruses block apoptosis, our understanding of the mechanisms by which these viruses trigger the apoptotic response lags behind. Attachment to cell receptors is not sufficient to induce apoptosis, since blocking p35 mutant virus entry with chloroquine does not result in apoptosis ${ }^{2}$ and UVinactivated virus also does not induce apoptosis (Clem RJ and Miller LK, unpublished results). Therefore the death trigger involves some step subsequent to virus attachment.

Once inside the cell, baculovirus replication can be broken down into early, late, and very late phases. The key event that regulates transition from the early into the late phase is the onset of viral DNA replication. Treatment with an inhibitor of DNA synthesis blocks entry into the late phase and also blocks apoptosis of SF-21 cells infected with an AcMNPV mutant lacking the p35 gene. ${ }^{3}$ This result suggests that the initiation of apoptosis is either dependent on a late viral gene product or is due to some other process associated with the transition from the early to late stages of infection. Since there are several interrelated processes occurring at this crucial time, it is difficult to determine precisely what is responsible for the induction of apoptosis. These processes include: (1) the initiation of viral DNA synthesis, (2) the initiation of late viral gene expression, and (3) the cessation of synthesis of cellular RNA and protein, along with that of early viral gene products. Confounding the situation is the fact that there is evidence for more than one of these processes contributing to the induction of apoptosis (Figure 1). For example, simply blocking RNA synthesis is sufficient to induce apoptosis in SF-21 cells. ${ }^{3}$ Treatment with any of several different RNA synthesis inhibitors including actinomycin D, 5,6-dichlorobenzimidazole riboside, or $\alpha$-amanatin induces rapid apoptosis in these cells. ${ }^{3}$ Thus the shut-off of host mRNA synthesis seen at late times in AcMNPV infection could be responsible for the induction of apoptosis, ${ }^{3}$ presumably by preventing the synthesis of an inhibitory protein that is required to prevent the initiation of death in these cells.

The induction of DNA synthesis itself may also be what triggers the apoptotic death of AcMNPV-infected SF-21 cells. The process of viral DNA replication has been studied by transiently expressing the set of viral genes necessary for DNA synthesis. ${ }^{4}$ In these assays, very little DNA can be recovered unless $p 35$ or an active iap gene such as Op-iap or Cp-iap is included in the transfections, suggesting that apoptosis is triggered by expression of the viral DNA synthesis machinery. ${ }^{4}$ One of the genes required for viral DNA replication is ie-1, which encodes a transcriptional activator protein that also appears to play a role in DNA replication. Expression of ie-1 itself has been shown to induce apoptosis, ${ }^{5}$ and its ability to do so is enhanced by another baculovirus gene, pe38. ${ }^{6}$ The predicted PE38 protein contains an amino terminal nuclear localization signal and a leucine zipper motif, which are both required for enhancement of ie-1-induced apoptosis, and a RING motif, which is not required. ${ }^{6}$ Both ie-1 and pe38 are expressed throughout AcMNPV infection, from immediately after infection to the late phase, and both genes encode transcription factors. Since ie-1 is expressed at both early and late times, it is expressed in aphidicolin-treated cells. This suggests that ie-1 induces apoptosis through the initiation of DNA synthesis, since aphidicolin blocks AcMNPV-induced apoptosis but does not block expression of ie-1.

Of course, it may be that more than one process (either cessation of RNA synthesis or induction of viral DNA

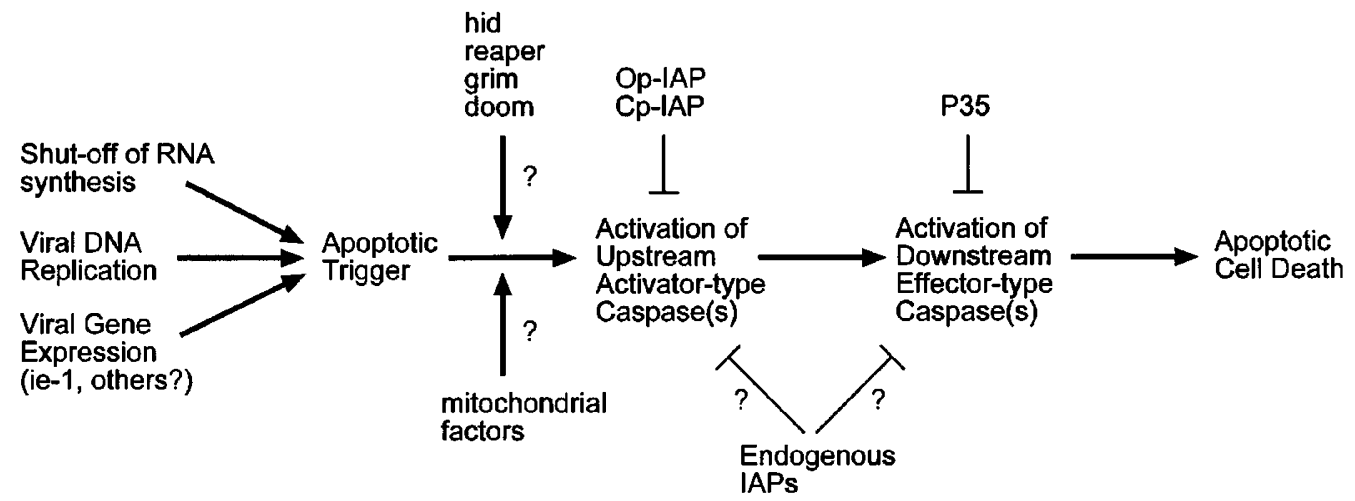

Figure 1 Pathways of baculovirus-induced apoptosis in SF-21 cells. There appear to be at least three different baculovirus-induced apoptotic stimuli that can contribute to the triggering of apoptosis in SF-21 cells: shutting of cellular RNA synthesis, viral DNA replication, and expression of pro-apoptotic viral genes such as ie-1 and pe38. Currently, it is not known whether lepidopteran homologs of hid, reaper, grim, and doom are involved in baculovirus-induced apoptosis. The role of mitochondrial factors such as cytochrome $c$ in lepidopteran apoptosis is also unknown. The baculovirus Op-IAP protein appears to act either by preventing the activation of upstream activator caspase(s) or by directly inhibiting the upstream caspase(s) themselves, while baculovirus P35 prevents the activation of downstream effector caspases but is unable to block upstream caspases, as evidenced by its inability to prevent the initial processing of the downstream caspases Sf-caspase 1. Recent evidence suggests that Cp-IAP and endogenous Sf-IAP can inhibit a mammalian upstream caspase, ${ }^{34}$ but no information is available concerning their role in SF-21 cells 
synthesis) is involved in inducing apoptosis in AcMNPVinfected SF-21 cells. Additional evidence for more than one apoptosis-inducing function during AcMNPV infection comes from a study using a temperature sensitive mutant of AcMNPV that was defective in DNA synthesis. ${ }^{2}$ In this study, apoptosis was observed at the non-permissive temperature when viral DNA synthesis was blocked, suggesting that early functions were sufficient to induce apoptosis. However, a temperature-sensitive late function was required for maximal induction of apoptosis. The authors concluded that there were both early and late components involved in the induction of apoptosis. ${ }^{2}$ The early function may be expression of the ie-1 and pe38 genes and/or other early viral gene products.

Although not involved in inducing apoptosis, another player in AcMNPV-induced cell death is a gene called $p 10$. AcMNPV mutants lacking a functional $p 10$ gene but containing functional p35 exhibit a phenotype in which the nucleus fails to lyse at late times. ${ }^{7}$ Although the death of cells infected with p35-containing viruses does not appear to be apoptotic, studies with adenovirus suggest there may be a role for $p 10$ in the late lysis of cells infected with AcMNPV. The E3 region of adenovirus encodes a small (11.6 kDa) glycoprotein called adenovirus death protein or ADP that localizes in the nuclear membrane at late times in adenovirus-infected cells. ${ }^{8}$ Adenovirus mutants lacking ADP exhibit a phenotype similar to that of $p 10$ mutants of AcMNPV, in which the nucleus fails to lyse. Although P10 and ADP do not share any significant sequence homology, both proteins are small, highly basic, and localized in the nucleus.

\section{The bad: how do baculoviruses block apoptosis?}

Like many other viruses, baculoviruses have acquired antiapoptotic genes during their evolution. The importance of these genes is borne out by the fact that the genomes of several baculoviruses have been sequenced, and so far, every one contains at least one homolog of an anti-apoptotic gene. To data, two different families of baculovirus antiapoptotic genes have been identified, p35 and iap.

\section{The p35 gene}

The p35 gene was first identified in 1987 by sequence analysis of a region of the AcMNPV genome adjacent to the insertion of a transposable element, ${ }^{9}$ but the function of the p35 gene product (P35) was unknown until the mapping of the spontaneous mutation in the annihilator mutant in $1991 .{ }^{1}$ Subsequent work showed that the P35 protein is a direct inhibitor of caspase ${ }^{10,11}$ and that it acts in a stoichiometric fashion as a suicide inhibitor. ${ }^{10}$ Cleavage of P35 by caspase is necessary but not sufficient for inhibitory function. ${ }^{12}$ Recently, the crystal structure of the AcMNPV P35 protein was solved, revealing a novel and highly interesting protease inhibitor structure. ${ }^{13}$ The overall structure of P35 is similar to a teapot, with a flexible, solvent-exposed 'handle' or loop that contains a caspase cleavage site at the apex, where it is accessible by caspases. Cleavage of the loop by caspase occurs following an aspartate residue, yielding cleavage products of 10 and $25 \mathrm{kDa}$ that remain stably associated with the caspase. Immediately below the reactive site loop in the structure is an $\alpha$-helix, and below that an eight-stranded $\beta$ sheet. Disruption of the $\alpha$-helix or the interaction between the $\alpha$-helix and the $\beta$-sheet by mutagenesis eliminated antiapoptotic function, and converted P35 from a stoichiometric caspase inhibitor into a caspase substrate. ${ }^{14}$ These results indicate that structural elements outside of the reactive site loop are involved in the inhibition mechanism.

To date, there are no known cellular homologs of the p35 gene. However, in addition to AcMNPV, two other baculoviruses have been found to carry p35-homologous genes, Bombyx mori NPV and Spodoptora littoralis NPV. The protein encoded by the BmNPV p35 gene is $90 \%$ identical to AcMNPV P35 at the amino acid level and also plays a role in preventing apoptosis induced by BmNPV in B. mori cells. ${ }^{15}$ The SINPV p35-homologous gene, p49, is more divergent, encoding a protein with $49 \%$ amino acid identity to AcMNPV $p 35$ and a predicted size of $49 \mathrm{kDa}^{16}$ Further investigation into this novel class of protease inhibitors promises to reveal interesting new information on biochemical mechanisms of protease inhibition, and may lead to new therapeutics.

Interestingly, there have been recent reports of the first caspase that is resistant to P35, the Drosophila caspase DRONC. ${ }^{17,18}$ DRONC is thought to be an upstream, activator-type caspase because of its long pro-domain. The observation that there are upstream caspases in insects that are resistant to P35 makes sense given the fact that P35 cannot block the initial processing steps of the downstream lepidopteran effector caspase Sf-caspase-1. ${ }^{19}$ Thus, in SF-21 cells, P35 appears to act by blocking the final processing and activation of downstream effector caspases (Figure 1).

\section{The iap gene family}

The first iap gene, $\mathrm{Cp}$-iap, was discovered in the baculovirus Cydia pomonella granulovirus using a genetic screen based on rescue of the annihilator mutant. ${ }^{20}$ To date, at least 10 different baculoviruses are known to contain one or more iaphomologous genes, although so far only $\mathrm{Cp}$-iap and Op-iap (from Orgyia pseudotsugata NPV) have been shown to have anti-apoptotic function. A large number of cellular iaphomologous genes have subsequently been reported in organisms ranging from yeast to humans.

IAP proteins are metalloproteins. The defining characteristic of IAP proteins is that they contain one or more copies of a zinc-binding motif called a BIR (Baculovirus IAP Repeat) near the amino terminus. In addition, many IAPS contain another type of zinc-binding motif called a RING domain at the carboxyl terminus. An individual BIR is approximately 70 amino acids in length and has a conserved Cys $\mathrm{X}_{2}$ Cys motif near its center followed by a His $\mathrm{X}_{6}$ Cys motif near its carboxyl end (where $X$ is any amino acid). ${ }^{21}$ There are also several other residues that are invariant in all known BIRs, as well as a number of other highly conserved residues. The solution structures of two BIR motifs have been recently reported, and they reveal that each BIR coordinates a single atom of zinc 
via the four conserved cysteine and histidine residues in a novel type of fold. ${ }^{22,23}$

BIR-containing portions of different IAP proteins have been shown to bind to a wide variety of different pro-death proteins, including the Drosophila proteins REAPER, GRIM, HID, and DOOM, components of the tumor necrosis factor signaling complex, and various caspases. $^{24}$ In addition to the importance of the BIRs themselves, the sequences flanking the BIRs may also play important roles in determining the binding partners and in anti-apoptotic function. Deletion analysis of Op-IAP revealed that the region responsible for binding HID was at the C-terminal end of BIR2. ${ }^{25}$ Also, the region flanking the amino terminal end of BIR2 of XIAP was found to be important for caspase inhibition. ${ }^{23}$ Single BIRs from XIAP, Op-IAP, and DIAP1 have been shown to be sufficient for anti-death activity, ${ }^{25,26}$ although in the case of Op-IAP, the anti-apoptotic function of a single BIR is greatly reduced compared to that of the full length protein. ${ }^{25}$

There is mounting evidence that at least a subset of IAP proteins function as direct caspase inhibitors. The first IAP protein to be shown to inhibit caspases directly was XIAP, and subsequently cIAP-1, CIAP-2, and DIAP1 have also been shown to be capable of inhibiting certain caspases. $^{27}$ Genetic and biochemical evidence was recently published suggesting that DIAP1 is normally bound to and inhibiting one or more of the Drosophila caspases, and that the function of HID is to bind DIAP1 and release the active caspase. ${ }^{28,29}$ At least for DIAP1, these results apparently resolve the question of whether IAPs act by inhibiting the function of proteins like HID, REAPER, and GRIM, or whether it is the other way around. The genetic evidence in Drosophila strongly indicates that DIAP1 functions downstream of HID, REAPER, and GRIM in developmentally programmed cell death, favoring the model that these pro-death molecules inhibit the ability of DIAP1 to inhibit caspases. However the situation may be different in other death paradigms, such as irradiation-induced apoptosis. One could easily imagine alternative scenarios where upregulation of DIAP1 or other IAPs could result in titration of REAPER, HID or GRIM by excess IAPs. Since the baculovirus IAPs Op-IAP and Cp-IAP also bind HID, REAPER, and GRIM, they may work in a fashion similar to that of DIAP1. However, the fact that the RING is required for optimal anti-death function of the baculovirus IAPs but not of cellular IAPs suggests that we may still encounter some surprises in determining the mode of action of the baculovirus IAPS.

In SF-21 cells, the baculovirus Op-IAP protein acts at a point upstream of $P 35,,^{19,30,31}$ either by preventing the activation of or by directly inhibiting an as yet unidentified upstream caspase (Figure 2). In support of this, evidence was recently published suggesting that Cp-IAP directly inhibits a mammalian activator caspase, caspase $-9^{32}$. Puzzling, however, was the finding that Op-IAP does not inhibit caspase $-9^{32}$. Although Drosophila HID, REAPER, GRIM, and DOOM all induce apoptosis when overexpressed in SF-21 cells, and all of these proteins bind Op-IAP, it is not known yet whether the SF-21 homologs of these proteins are involved in triggering baculovirusinduced apoptosis in SF-21 cells.

Evidence has also been published in the past year suggesting that oligomerization of Op-IAP is mediated by the BIRs and is required for its function. ${ }^{33} \mathrm{~A}$ truncated form of Op-IAP lacking the RING interfered with the function of the full length protein, and was shown to immunoprecipitate full length Op-IAP. The truncated form also sensitized SF21 cells to apoptosis presumably by interfering with endogenous IAPs, consistent with an earlier report. ${ }^{34}$ This opens up the possibility that homo-and hetero-complexes of IAPs may exist in cells, a notion that could have important implications for controlling binding specificity and apoptotic regulation by IAPs.

Two lepidopteran iap genes were reported in the past year, from Spodoptera frugiperda and Trichoplusia ni. ${ }^{32,35}$ Both of these insects are permissive hosts for AcMNPV. Interestingly, these IAP proteins resemble the baculovirus IAPs more closely than other cellular IAPs, including those from Drosophila. Thus it seems probable that the baculoviruses have pirated IAP proteins from their lepidopteran hosts.

Recently, evidence has come to light indicating that some proteins with BIR motifs have functions other than blocking apoptosis, which has led to the proposal of the name BIR-containing proteins or BIRPs. ${ }^{36}$ Here, I will use this name to refer to BIR-containing proteins that do not have apparent anti-apoptotic activity. BIRPs from nematode and yeast appear to be important in regulating cytokinesis, and have no apparent anti-apoptotic function. ${ }^{37-40}$ The yeast and nematode BIRPs are most similar to survivin, a human protein containing a single BIR and no RING. Interestingly, survivin can regulate both apoptosis and cytokinesis. ${ }^{41}$ This raises the interesting possibility that the ancestral function of IAP proteins may have been in regulating cell division, and that a role in apoptosis may have arisen later in evolution.

It has been known for some time that the viral IAP homolog found in AcMNPV (Ac-iap or Ac-iapl) does not have any apparent anti-apoptotic activity. ${ }^{3}$ Recent results indicate, however, that deletion of Ac-iapl from the AcMNPV genome provides a species-specific replication advantage for the virus (McLachlin JR et al., manuscript submitted). Although the function of Ac-iapl is still not clear, these results suggest that it does have a function of some kind, and that this function must be important for the virus in order for this gene to have been retained throughout evolution despite the fact that its presence retards viral replication in at least one insect species.

As mentioned above, many IAP proteins also contain a type of zinc finger known as a RING motif at the carboxyl terminus. The RING (or $\mathrm{C}_{3} \mathrm{HC}_{4}$ ) motif was first described in $1991,{ }^{42}$ and is now known to be present in more than 200 proteins. These proteins are involved in a variety of functions, which at first do not seem to have anything in common. However, upon closer examination, the common theme in RING function appears to be their involvement in 'scaffolding' or building multi-protein complexes. ${ }^{43}$ Each RING motif is capable of binding two atoms of zinc and forming a highly stable platform on which molecular 
A<smiles>C1CCC(C2CCCC2)C1</smiles>

T. ni

T. ni
B

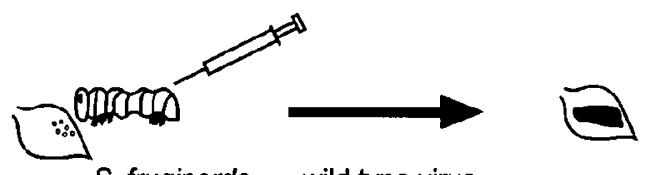

S. frugiperda wild type virus
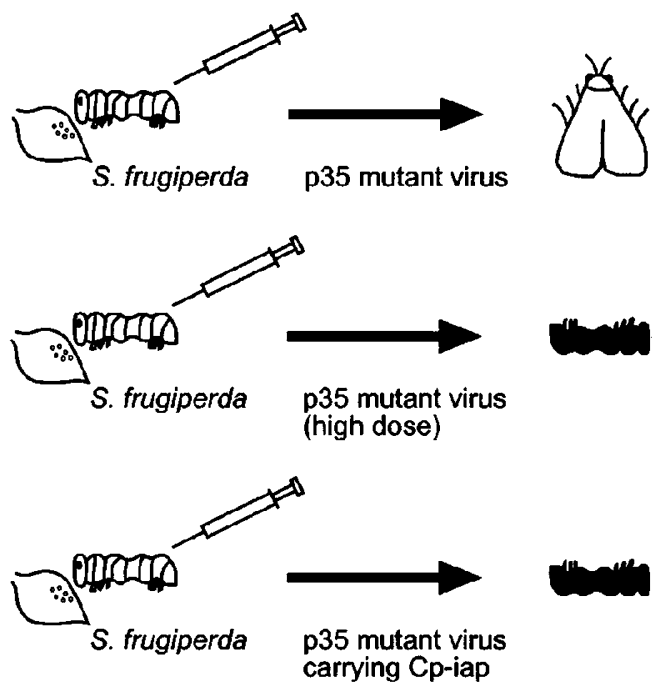

Figure 2 The effects of the anti-apoptotic genes p35 and iap on baculovirus pathogenesis. In natural situations, the lepidopteran insects Trichoplusia ni (the cabbage looper) and Spodoptera frugiperda (the fall armyworm) become infected when larvae feed on leaves contaminated with occluded virus. In the laboratory, larvae can also be experimentally infected by injection of budded virus. (A) In T. ni, wild-type virus kills and liquefies the insect (as illustrated by the puddle of black liquid on the leaf), while larvae infected with virus mutants lacking the anti-apoptotic p35 gene still become melanized and die, but do not liquefy. (B) In $S$. frugiperda, wild-type virus kills and liquefies the larvae, but insects infected with doses of $p 35$ mutant virus sufficient to kill $T$. ni larvae pupate and survive to adulthood. This lack of infectivity can be overcome by infecting with extremely high doses of $p 35$ mutant virus or by using a virus carrying the Cp-iap gene, but in these cases the larvae do not liquefy

scaffolds or complexes can be assembled. One of the more interesting functions of RING motifs that has come to light recently is their involvement in the ubiquitination of proteins. Several RING-containing proteins have been shown to be components of E3 ubiquitin ligases. ${ }^{44}$ Indeed, some of the IAP proteins themselves have been shown to be capable of directing their own ubiquitination, and this activity requires the RING motif. ${ }^{45}$ Additional in vitro evidence suggests that clAP-1 can direct the ubiquitination of certain caspases. ${ }^{46}$ The relevance of this activity to in vivo IAP anti-apoptotic function is not yet clear, but this promises to be an exciting area of future study.

\section{The ugly: how does apoptosis affect baculovirus pathogenesis?}

Despite significant advances in our understanding of the insect immune system and how it combats fungal and bacterial pathogens, there is little evidence to date on how insects defend themselves against viruses. The role of apoptosis in anti-viral defense in lower animals has been suspected for over a decade, ${ }^{47}$ but the best evidence that apoptosis can be a significant anti-viral defense comes from work done with baculovirus mutants that cannot block apoptosis.
Baculoviruses come in two forms, the budded virus, which consists of enveloped nucleocapsids and serves to spread infection within an infected host, and the occluded virus, which consists of enveloped nucleocapsids embedded in large protein crystals that serve to protect the virus when it is outside of the host in the environment and allow spread of infection from one host to another. The larvae of lepidopteran insects (commonly known as caterpillars) can be infected either by injection of budded virus into the hemocoel (blood), or by feeding occluded virus to the insects, although the normal route of infection in nature is by feeding (Figure 2). Baculoviruses encode proteins, such as chitinases and proteases, that aid in the disintegration of the insect cadaver. Thus, at the late stages of infection, a baculovirus-infected insect liquefies, or 'melts', into a puddle of black slime (the ugly part of our narrative).

AcMNPV normally causes lethal disease in a number of insect species, including both $S$. frugiperda and $T$. ni. Cell lines derived from these species differ in their response to infection with mutants of AcMNPV that lack the p35 gene. ${ }^{48,49}$ SF-21 cells, derived from $S$. frugiperda, undergo apoptosis when infected with p35 mutant viruses, and the yields of both budded and occluded virus are drastically reduced. TN-368 cells, derived from $T$. ni, do not undergo 
apoptosis and instead appear identical to cells infected with wild type AcMNPV, with normal yields of budded and occluded virus.

When p35 mutants are used to infect larvae of these species, a correlation is observed between induction of apoptosis in the cell lines and a reduction in infectivity in the host ${ }^{48,50}$ (Figure 2). In $S$. frugiperda, the dose of mutant virus required to kill $50 \%$ of the larvae is increased 1000 fold by the injection route and 25 -fold by the oral route compared to the wild-type virus. In T. ni larvae, the dose of p35 mutant virus required to kill $50 \%$ of the larvae is essentially the same as that of wild-type virus by either route, although $T$. ni larvae infected by injection of p35 mutant virus do not liquefy. The basis for the lack of liquefaction is not understood. It may be that p35 is required for successful infection of certain tissues in the insect critical for liquefaction to occur.

If a large enough dose of p35 mutant virus is administered, $S$. frugiperda larvae can still be infected and the infection proceeds normally (although again, the insects do not liquefy) ${ }^{48}$ (Figure 2). Additional evidence that apoptosis is responsible for the reduction in infectivity in $S$. frugiperda is that the infectivity is restored to normal when a recombinant virus carrying $\mathrm{Cp}$-iap instead of $p 35$ is used, although $\mathrm{Cp}$-iap does not restore liquefaction, ${ }^{50}$ suggesting that $p 35$ and $\mathrm{Cp}$-iap may not be completely functionally interchangeable (Figure 2).

Thus there is an excellent correlation between the ability of the virus to block apoptosis in cell culture and its ability to productively infect insect larvae. The observation in $S$. frugiperda that infectivity can be achieved by infecting with a larger dose of $p 35$ mutant virus indicates that the role of apoptosis is in preventing the establishment of the infection at the early stages. If the initially infected cells undergo apoptosis, this may allow the host to clear the infection, perhaps by engulfment or encapsulation of infected cells. However, if the virus prevents apoptosis, the infection may proceed too quickly for the host defenses to be successful. The same effect may be obtained by injecting a large enough dose of p35 mutant virus. However, at this point, these are speculations, since we have no direct evidence pertaining to what is going on within the infected insect.

\section{Conclusions and perspectives}

It has been an exciting decade since the initial discovery that baculoviruses have the ability to manipulate the apoptotic response of their insect hosts. Much progress has occurred in the determination of how the viral proteins P35 and IAP and their cellular counterparts actually function at the biochemical level in blocking apoptosis. We have also made significant inroads into improving our understanding of how an apoptotic response can be an effective anti-viral defense. But as with all good scientific endeavors, as we continue to obtain exciting new data, more and more questions are opening up. How do baculoviruses induce apoptosis? What role, if any, do homologs of Drosophila death genes and mitochondrial factors play in baculovirus-induced cell death? What is the biochemical mechanism of caspase inhibition by P35? Are all IAPs simply caspase inhibitors, or is the story more complicated? Is the mode of action of baculovirus IAP proteins similar to that of cellular IAPs, given the differences in the requirement for the RING motif between the two types of proteins? What is the role of ubiquitination in the function of IAP proteins? What is the importance of oligomerization of IAPs, and can the ability to form heteromeric IAP complexes regulate their activity? What is the function of viral IAPs like Ac-IAP1 that don't appear to block apoptosis? Can apoptosis serve as an anti-viral defense mechanism? If so, how widespread is it? How does apoptosis influence the in vivo replication and pathogenesis of baculoviruses? What are the important tissues involved in the insect? The next decade of research on baculoviruses and apoptosis promises to answer many of these questions.

\section{Acknowledgements}

This review is dedicated to the memory of the late $\mathrm{Dr}$. Lois K. Miller: mentor, teacher, friend, and above all, scientist par excellence. I thank Dr. A. Lorena Passarelli for helpful comments on the manuscript.

\section{References}

1. Clem RJ, Fechheimer M and Miller LK (1991) Prevention of apoptosis by a baculovirus gene during infection of insect cells. Science 254: 1388-1390

2. LaCount DJ and Friesen PD (1997) Role of early and late replication events in induction of apoptosis by baculoviruses. J. Virol. 71: 1530-1537

3. Clem RJ and Miller LK (1994) Control of programmed cell death by the baculovirus genes p35 and iap. Molec. Cell. Biol. 14: 5212-5222

4. Lu A and Miller LK (1995) The roles of eighteen baculovirus late expression factor genes in transcription and DNA replication. J. Virol. 69: 975-982

5. Prikhod'ko EA and Miller LK (1996) Induction of apoptosis by baculovirus transactivator IE1. J. Virol. 70: 7116-7124

6. Prikhod'ko EA and Miller LK (1999) The baculovirus PE38 protein augments apoptosis induced by transactivator IE1. J. Virol. 73: 6691-6699

7. van Oers MM, Flipsen JTM, Reusken CBEM, Sliwinsky EL, Goldbach RW and Vlak JM (1993) Functional domains of the p10 protein of Autographa californica nuclear polyhedrosis virus. J. Gen. Virol. 74: 563-574

8. Tollefson AE, Scaria A, Hermiston TW, Ryerse JS, Wold LJ and Wold WSM (1996) The adenovirus death protein (E3-11.6K) is required at very late stages of infection for efficient cell lysis and release of adenovirus from infected cells. J. Virol. 70: 2296-2306

9. Friesen PD and Miller LK (1987) Divergent transcription of early 35 and 94kilodalton protein genes encoded by the Hind-III K genome fragment of the baculovirus Autographa californica nuclear polyhedrosis virus. J. Virol. 61: 2264-2272

10. Bump NJ, Hackett M, Hugunin M, Seshagiri S, Brady K, Chen P, Ferenz C, Franklin S, Ghayur T, Li P, Licari P, Mankovich J, Shi L, Greenberg AH, Miller LK and Wong WW (1995) Inhibition of ICE family proteases by baculovirus antiapoptotic protein p35. Science 269: $1885-1888$

11. Xue D and Horvitz HR (1995) Inhibition of the Caenorhabditis elegans cell-death protease CED-3 by a CED-3 cleavage site in baculovirus p35 protein. Nature (London) 377: 248-251

12. Bertin J, Mendrysa SM, LaCountDJ, Gaur S, Krebs JF, Armstrong RC, Tomaselli KJ and Friesen PD (1996) Apoptotic suppression by baculovirus P35 involves cleavage by and inhibition of a virus-induced CED-3/ICE-like protease. J. Virol. 70: 6251-6259

13. Fisher AJ, dela Cruz W, Zoog SJ, Schneider CL and Friesen PD (1999) Crystal structure of baculovirus P35: role of a novel reactive site loop in apoptotic caspase inhibition. EMBO J. 18: 2031-2039

14. Zoog SJ, Bertin J and Friesen PD (1999) Caspase inhibition by baculovirus P35 requires interaction between the reactive site loop and the beta-sheet core. J. Biol. Chem. 274: 25995-26002 
15. Kamita SG, Majima K and Maeda S (1993) Identification and characterization of the p35 gene of Bombyx mori nuclear polyhedrosis virus that prevents virusinduced apoptosis. J. Virol. 67: 455-463

16. Du Q, Lehavi D, Faktor O, Qi Y and Chejanovsky N (1999) Isolation of an apoptosis suppressor gene of the Spodoptera littoralis nucleopolyhedrovirus. J. Virol. 73: 1278-1285

17. Hawkins CJ, Yoo SJ, Peterson EP, Wang SL, Vernooy SY and Hay BA (2000) The Drosophilacaspase DRONC cleaves following glutamate or aspartate and is regulated by DIAP1, HID, and GRIM. J. Biol. Chem. 275: 27084-27093

18. Meier P, Silke J, Leevers SJ and Evan GI (2000) The Drosophila caspase DRONC is regulated by DIAP1. EMBO J. 19: $598-611$

19. LaCount DJ, Hanson SF, Schneider CL and Friesen PD (2000) Caspase inhibitor P35 and inhibitor of apoptosis Op-IAP block in vivo proteolytic activation of an effector caspase at different steps. J. Biol. Chem. 275: 15657-15664

20. Crook NE, Clem RJ and Miller LK (1993) An apoptosis-inhibiting baculovirus gene with a zinc finger-like motif. J. Virol. 67: 2168-2174

21. Clem RJ, Hardwick JM and Miller LK (1996) Anti-apoptotic genes of baculoviruses. Cell Death Differ. 3: 9-16

22. Hinds MG, Norton RS, Vaux DL and Day CL (1999) Solution structure of a baculoviral inhibitor of apoptosis (IAP) repeat. Nature Struct. Biol. 6: 648-651

23. Sun C, Cal M, Gunasekera AH, Meadows RP, Wang H, Chen J, Zhang H, Wu W $\mathrm{Xu} \mathrm{N}, \mathrm{Ng} \mathrm{S}-\mathrm{C}$ and Fesik SW (1999) NMR structure and mutagenesis of the inhibitor-of-apoptosis protein XIAP. Nature (London) 401: 818-822

24. Miller LK (1999) An exegesis of IAPs: salvation and surprises from BIR motifs. Trends Cell Biol. 9: 323-328

25. Vucic D, Kaiser WJ and Miller LK (1998) A mutational analysis of the baculovirus inhibitor of apoptosis Op-IAP. J. Biol. Chem. 273: 33915-33921

26. Takahashi R, Deveraux Q, Tamm I, Welsh K, Assa-Munt N, Salvesen GS and Reed JC (1998) A single BIR domain of XIAP sufficient for inhibiting caspases. J. Biol. Chem. 273: $7787-7790$

27. Deveraux QL and Reed JC (1999) IAP family proteins-suppressors of apoptosis. Genes Dev. 13: 239-252

28. Goyal L, McCall K, Agapite J, Hartwieg E and Steller H (2000) Induction of apoptosis by Drosophila reaper, hid and grim through inhibition of IAP function. EMBO J. 19: $589-597$

29. Wang SL, Hawkins CJ, Yoo SJ, Muller H-AJ and Hay BA (1999) The Drosophila caspase inhibitor DIAP1 is essential for cell survival and is negatively regulated by HID. Cell. 98: 453-463

30. Manji GA, Hozak RR, LaCount DJ and Friesen PD (1997) Baculovirus inhibitor of apoptosis functions at or upstream of the apoptotic suppressor P35 to prevent programmed cell death. J. Virol. 71: 4509-4516

31. Seshagiri S and Miller LK (1997) Baculovirus inhibitors of apoptosis (IAPs) block activation of Sf-caspase-1. Proc. Natl. Acad. Sci. USA 94: 13606-13611

32. Huang Q, Deveraux QL, Maeda S, Salvesen GS, Stennicke HR, Hammock BD and Reed JC (2000) Evolutionary conservation of apoptosis mechanisms: Lepidopteran and baculoviral inhibitor of apoptosis proteins are inhibitors of mammalian caspase-9. Proc. Natl. Acad. Sci. USA 97: 1427-1432

33. Hozak RR, Manji GA and Friesen PD (2000) The BIR motifs mediate dominant interference and oligomerization of inhibitor of apoptosis Op-IAP. Molec. Cell. Biol. 20: 1877-1885
34. Harvey AJ, Soliman H, Kaiser WJ and Miller LK (1997) Anti- and pro-apoptotic activities of baculovirus and Drosophila IAPs in an insect cell line. Cell Death Differ. 4: 733-744

35. Seshagiri S, Vucic D, Lee J and Dixit VM (1999) Baculovirus-based genetic screen for antiapoptotic genes identifies a novel IAP. J. Biol. Chem. 274:36769 36773

36. Uren AG, Coulson EJ and Vaux DL (1998) Conservation of baculovirus inhibitor of apoptosis repeat proteins (BIRPs) in viruses, nematodes, vertebrates and yeasts. Trends Biochem. Sci. 23: 159-162

37. Fraser AG, James C, Evan Gl and Hengartner MO (1999) Caenorhabditis elegans inhibitor of apoptosis protein (IAP) homologue BIR-1 plays a conserved role in cytokinesis. Curr. Biol. 9: 292-301

38. Li F, Flanary PL, Altieri DC and Dohlman HG (2000) Cell division regulation by BIR1, a member of the inhibitor of apoptosis family in yeast. J. Biol. Chem. 275 $6707-6711$

39. Uren AG, Beilharz T, O'Connell MJ, Bugg SJ, van Driel R, Vaux DL and Lithgow T (1999) Role for yeast inhibitor of apoptosis (IAP)-like proteins in cell division. Proc. Natl. Acad. Sci. USA 96: 10170-10175

40. Yoon H-J and Carbon J (1999) Participation of Bir1p, a member of the inhibitor of apoptosis family, in yeast chromosome segregation events. Proc. Natl. Acad. Sci. U.S.A. 96: $13208-13213$

41. Li F, Ambrosini G, Chu EY, Plescia J, Tognin S, Marchisio PC and Altieri DC (1998) Control of apoptosis and mitotic spindle checkpoint by survivin. Nature (London) 396: 580-584

42. Freemont PS, Hanson IM and Trowsdale J (1991) A novel cysteine-rich sequence motif. Cell. 64: 483-484

43. Borden KLB (2000) RING domains: master builders of molecular scaffolds? J. Mol. Biol. 295: 1103-1112

44. Tyers M and Willems AR (1999) One RING to rule a superfamily of E3 ubiquitin ligases. Science. 284:601-604

45. Yang Y, Fang S, Jensen JP, Weissman AM and Ashwell JD (2000) Ubiquitin protein ligase activity of IAPs and their degradation in proteasomes in response to apoptotic stimuli. Science. 288: 874-877

46. Huang H-K, Joazeiro CAP, Bonfoco E, Kamada S, Leverson JD and Hunter T (2000) The inhibitor of apoptosis, clAP2, functions as a ubiquitin-protein ligase and promotes in vitromonoubiquitination of caspases 3 and 7 . J. Biol. Chem. 275 26661-26664

47. Clouston WM and Kerr JFR (1985) Apoptosis, lymphocytotoxicity and the containment of viral infections. Med. Hypoth. 18: 399-404

48. Clem RJ and Miller LK (1993) Apoptosis reduces both the in vitro replication and the in vivo infectivity of a baculovirus. J. Virol. 67: 3730-3738

49. Hershberger PA, Dickson JA and Friesen PD (1992) Site-specific mutagenesis of the 35-kilodalton protein gene encoded by Autographa californica nuclear polyhedrosis virus: cell line-specific effects on virus replication. J. Virol. 66: $5525-5533$

50. Clem RJ, Robson M and Miller LK (1994) Influence of infection route on the infectivity of baculovirus mutants lacking the apoptosis-inhibiting gene $p 35$ and the adjacent gene p94. J. Virol. 68: 6759-6762 